\title{
COLITECVS - NEW TOOL FOR AUTOMATED REDUCTION OF PHOTOMETRIC OBSERVATIONS
}

\author{
V. E. Savanevych ${ }^{1}$, O. B. Briukhovetskyi ${ }^{1}$, S. V. Khlamov ${ }^{2}$, A. V. Pohorelov ${ }^{3}$, V. P. Vlasenko ${ }^{1}$, \\ P. A. Dubovský ${ }^{4}$, I. Kudzej ${ }^{4}, \breve{S}$. Parimucha ${ }^{5}$ \\ ${ }^{1}$ Western Radio Technical Surveillance Center, National Space Agency of Ukraine, \\ Mukachevo, Ukraine,vadym@savanevych.com, izumsasha@gmail.com \\ ${ }^{2}$ Uzhhorod National University, Laboratory of space research, \\ Uzhhorod, Ukraine,sergii.khlamov@gmail.com,colitec@neoastrosoft.com \\ ${ }^{3}$ Kharkiv National University of Radioelectronics, \\ Kharkiv, Ukraine, artempogorelov@gmail.com \\ ${ }^{4}$ Vihorlat Observatory, \\ Humenné, Slovakia,var@kozmos.sk,vihorlatobs1@stonline.sk \\ ${ }^{5}$ Faculty of Science, \\ UPJŠ Košice, Slovakia, stefan.parimucha@upjs.sk
}

ABSTRACT. The capabilities of telescopes allow us to make the plotting of light curves a routine task. This one shifts the main attention of astronomer from the plotting to research. To achieve this goal, we developed a new tool for automated reduction of photometric observations, which includes the computational method for the brightness assessment of the investigated and comparison stars; brightness equalization of astronomical images using inverse median filter; light curve plotting and its processing using different tools.

Keywords: CCD, photometric observations, light curve, star's brightness, reduction, inverse median filter.

\section{Introduction}

At the present time the astronomers have to perform a lot of actions to plot the light curve. This is caused by the fact that the programs for plotting light curves use only the images of objects indicated by the astronomer in the frame. Otherwise, there are areas of the astronomical researches, where the frames are processed completely, with assumption of the different parameters of all objects in frames. For example, the programs those realize the full processing of the asteroid surveys results and automatic detection for asteroids and comets in the series of CCDframes such as CoLiTec (Savanevych V. E. et al., 2015).

Authors combined existing methods of the light curves creation with the methods for full frames processing with the parameters estimation of all objects in these frames. Before the light curve plotting the anomaly pixels in CCD-frames are taken into account; the preliminary segmentation of celestial objects images is carried out as well as the assumption of the brightness and equatorial coordinates of objects with automatic selection of reference stars in the frame.

\section{Brightness equalization of astronomical images}

Astronomical digital images may be divided into coarse-grained and fine-grained components. Coarsegrained components correspond to the illumination of the image during astronomical observations at full moon or at sunrise/sunset and cover large part of frame. Fine-grained components correspond to the images of celestial objects. The size of them usually takes $5 \div 10$ pixels and no more than $50 \div 60$ pixels.

Coarse-grained components of the image correspond to low-frequency harmonics image spectrum, and finegrained components correspond to high-frequency harmonics. To remove coarse-grained components from the image it is possible to use a high-pass filter. Difference in sizes of objects images and background (coarse-grained components of astronomical images) allows using median filtering of the image for coarse-grained components extraction. To equalize the image background, the coarsegrained component obtained after median filtering is subtracted from the original image.

The inverse median alignment is used in conjunction with service frames. It is especially important to use a master-dark frame. All master-frames are formed pixel by pixel. $10 \%$ of the largest and $10 \%$ of the smallest values of brightness of the each pixel is tentatively discarded. For the remaining values of calibration frames the average and RMS are calculated. Brightness of the master-dark frame pixel is considered equal to the mean value of the pixel brightness only for pixels that do not differ from the mean by more than three RMS.

In contrast to the flat-calibration of frames, inverse median filter removes coarse-grained components that are caused by the illumination from the moon, the sun, and illuminations of anthropogenic origin. 
In this paper as the brightness equalization quality indicators we use the mean value and RMS of background brightness on a set of background pixels of the investigated local segment before and after operation of equalization. As a result of the brightness equalization the range of average background pixels brightness values has decreased in 131 times (quantiles of the indicated local means 0,95 and 0,05 differs in 131 times) for the segments of 30x30 pixels and in 145 times for the segments of $50 \times 50$ pixels. At the same time, the RMS of background pixels brightness in the investigated segments has not significantly changed. The value of quantiles of local RMS at the level of 0.95 decreases in 1.2 times in segments of $30 \times 30$ pixels and in 1.3 times for segments of 50x50 pixels.

\section{Computational method for the brightness assess- ment of the investigated star}

The computational method for the brightness assessment of the investigated star, comparisons stars and light curve creation includes the following operations.

1. Selection of the comparison stars by observer or automatically by proximity to investigated star by the attributes (distance, brightness and spectral class). Automatically selection is performed in two stages. At the first stage, selection of the stars, information about which is presented in APASS9 catalog and the image of which is single. On the second stage, sorting of the stars after first stage by the attributes. After that the first nearest stars are selected.

2. Introduction of the frame's window for every frame of series and determination of the average instrumental brightness and its RMS of every star in it:

$$
\begin{gathered}
\bar{m}_{\text {IRjn }}=\frac{1}{N_{W}} \sum_{n=1}^{N_{W}} m_{\text {IRjn }}, \\
\sigma_{j n}=\sqrt{\sum_{n=1}^{N_{W}}\left(m_{I R j n}\right)^{2} /\left(N_{W}-1\right)-\left(\sum_{n=1}^{N_{W}} m_{I R j n} / N_{W}-1\right)^{2}},
\end{gathered}
$$

where $n$ - CCD-frame's number, $j$ - star's number, $m_{\text {IRjn }}$ - reducted instrumental brightness of $j$-th comparison star in $n$-th frame, $N_{W}$ - frames number in assessment window of the average and RMS of reducted instrumental brightness of comparison star.

3. Exclusion of photometric measurements of comparison stars with anomaly error of instrumental brightness in frame:

$$
\sqrt{\left(m_{I R j n}-\bar{m}_{I R j n}\right)^{2}}>\gamma \sigma_{j n},
$$

where $\gamma=2 \div 4$ - proportionality coefficient.

4. Determination of the brightness of artificial comparison star (average instrumental brightness of all available comparison stars):

$$
m_{\text {synn }}=\frac{1}{N_{\text {reffsel }}} \sum_{j=1}^{N_{\text {reffsel }} m_{I j n},}
$$

where $m_{\text {synn }}$ - instrumental brightness of artificial comparison star in $n$-th frame, $N_{\text {reffsel }}$ - number of com- parison stars, $m_{I j n}-$ instrumental brightness of $j$-th comparison star in $n$-th frame.

5. Picking the pair of two or more comparison stars.

5.1. Determination of the reduction coefficient of the instrumental brightness of comparison star $\ell$-th pair of comparison stars for $n$-th frame:

$$
\eta_{\ell n}=\sum_{j=1}^{N_{\text {accept } \ell}} m_{I j n} / \sum_{j=1}^{N_{\text {accept } \ell}} m_{I B j},
$$

where $m_{I B j}$ - instrumental brightness of $j$-th comparison star in the base frame, $N_{\text {accep } \ell}$ - stars number in $\ell$-th pair of comparison stars.

5.2. Reduction of the instrumental brightness of comparison stars to the base frame:

$$
m_{I R j(\ell, n)}=\frac{m_{I j n}}{\eta_{\ell n}}
$$

where $m_{I R j(\ell, n)}$ - reducted instrumental brightness of $j$-th comparison star in $n$-th frame using $\ell$-th pair of comparison stars, $\eta_{\ell n}$ - reduction coefficient of the instrumental brightness from $n$-th frame to the base one using $\ell$-th pair of comparison stars.

5.3. Determination of the weight of every star in the current pair:

$$
v_{\ell j}=\frac{1}{v_{\ell} \sigma_{m \ell j}^{2}},
$$

where $v_{\ell j}$ - weight of $j$-th comparison star using $\ell$-th pair of comparison stars.

$$
\begin{gathered}
v_{\ell}=\sum_{j=1}^{N_{\text {accept } \ell}} \frac{1}{\sigma_{m \ell j}^{2}}, \\
\sigma_{m \ell j}^{2}=\frac{\sum_{j=1}^{N_{b e g}}\left(m_{I R j(\ell, n)}\right)^{2}}{N_{b e g}-1}-\left(\frac{\sum_{j=1}^{N_{b e g}} m_{I R j(\ell, n)}}{N_{b e g}-1}\right)^{2}
\end{gathered}
$$

- dispersion of the reducted instrumental brightness of $j$ th comparison star using $\ell$-th pair of comparison stars.

5.4. Determination of the star's brightness in each frame for the selected pair:

$$
\begin{gathered}
m_{I \beta \ell n}=\bar{m}_{I \beta \ell}+\frac{1}{v_{\Sigma \ell}} \sum_{j=1}^{N_{\text {accept } \ell}} v_{\ell j}\left(m_{I j n}-\bar{m}_{I j}\right), \\
v_{\Sigma \ell}=\sum_{j=1}^{N_{a c c e p t \ell}} v_{\ell j}, \\
m_{V S \ell n}=m_{I V S n}-m_{I \beta \ell n}+m_{c a t \beta}, \\
m_{\text {synln }}=m_{\text {synn }}-m_{I \beta \ell n}+m_{c a t \beta} .
\end{gathered}
$$

6. Picking the best pair of comparison stars by the minimum of brightness RMS of the artificial comparison star on frames: 


$$
\sigma_{\text {synh }}=\sqrt{\frac{\sum_{n=1}^{\text {Nbeg }}\left(m_{\text {synen }}\right)^{2}}{N_{\text {beg }}-1}-\left(\frac{\sum_{n=1}^{\text {Nbeg }} m_{\text {synn } n}}{N_{\text {beg }}-1}\right)^{2}},
$$

where $\sigma_{\text {syn } \ell}-$ brightness RMS of the artificial star using $\ell$-th pair of comparison stars, $m_{\text {syn } \ell n}$ - measured brightness of the artificial star in $n$-th frame using $\ell$-th pair of comparison stars, $N_{b e g}$ - number of the raw CCDframes, $m_{V S \ell n}$ - measured brightness of star in $n$-th frame using $\ell$-th pair of comparison stars, $m_{I V S n}-$ instrumental brightness of star in $n$-th frame, $m_{\text {cat } \beta}-$ brightness assessment of main comparison star from catalog, $m_{I \beta \ell n}$ - measured instrumental brightness of the main comparison star in $n$-th frame using $\ell$-th pair of comparison stars.

\section{CoLiTecVS workflow for the automatic data re- duction}

CoLiTec project created the universal system for the automatic data reduction-CoLiTecVS (Savanevych V. E. et al., 2015). This system allows astronomer creating the light curve of investigated variable star without manualdata handling between processing steps.

At first, astronomer with a telescope and specified software takes images of the interesting sky areas where the investigated variable stars are located. These raw images will be moderated before its processing. At this stage faulty and unsupported frames will be rejected by OLDAS system as soon as they are formed. This technique allows greatly speeding up the processing and provides user immediate notification of emerging issues. Also OLDAS determines the frames affiliation to the series and telescopes.

CoLiTecVS has the following workflow: forming the series of frames with the investigated variable star; brightness equalization of frames using master-frames and inverse median filter; assessment of the brightness of investigated star using the developed computational method; preparing the task-file with selected comparison stars; processing of the photometric observations; light curve creation of the investigated variable star. Created light curves can be viewed and analyzed using the specific modules of CoLiTecVS - PlotViewer or Virtual Observatory (VO).

\section{Representation of the photometric observations in CoLiTecVS}

PlotViewer is the local tool of CoLiTecVS for light curves viewing of the different variable stars and formed by the different telescopes. You can moderate these light curves by removing the faulty photometric observations of the investigated variable stars. Also you can change scale of the plot for the accurate investigation of photometric observations. It's important that the plot represents photometric observations even during the processing by updating plot when the new data arriving (fig. 1).

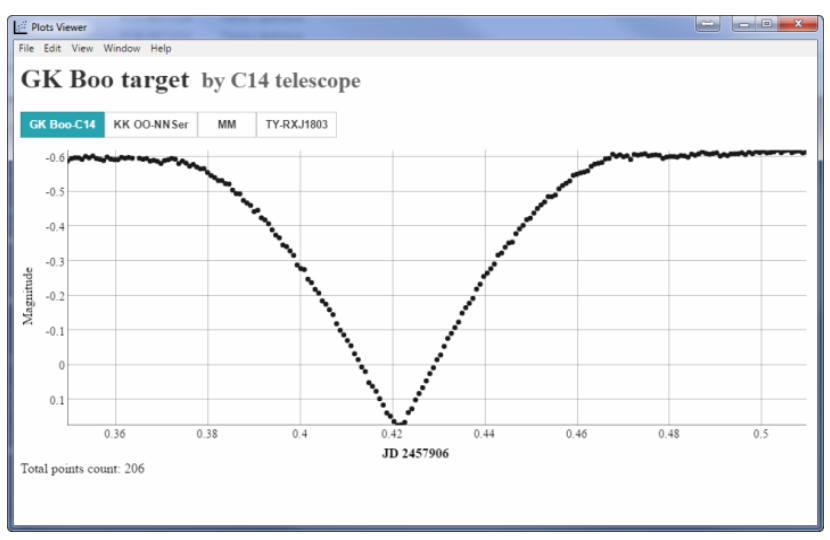

Figure 1: Light curve in PlotViewer

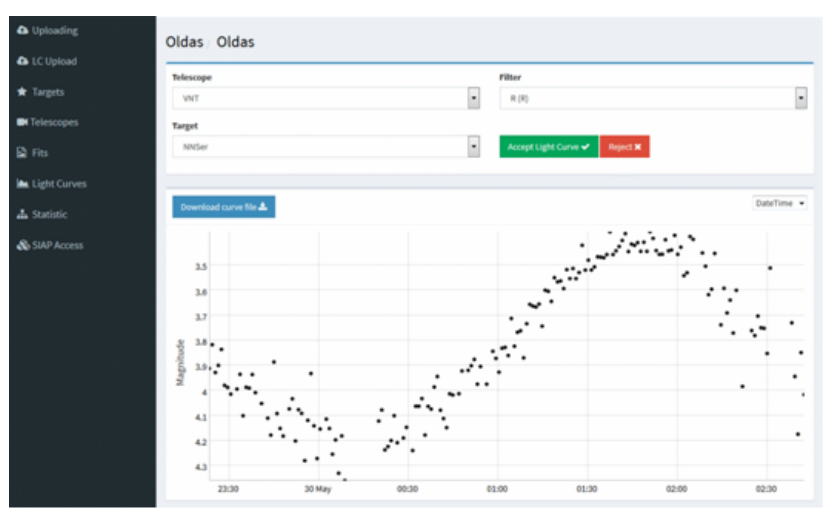

Figure 2: Light curve in VO

All processed photometric observations, light curves and even raw frames can be also sent to the Virtual Observatory (VO). These data are stored not only in the local database, but also published via SIAP protocol.

CoLiTecVS was designed according to IVOA recommendations for photometric data storing using crossreferences. This one allows receiving all relevant information such as required frames, light curves or photometric observations. VO web-interface represents light curves as a graph of brightness measurements variation. It is plotted by measurements from storage, data from different catalogs and telescopes (fig. 2). Detailed information about the appropriate magnitude and dimension of this frame is displayed after clicking on a point of the graph that represents the specific measurement.

Light curves of the investigated variable stars should be moderated and accepted via web interface of VO. On this stage astronomer may review and edit all received light curves and specified data (filter, target name, measurements, etc.). Also, familiarization with the attached notes can help researcher with the analyze of investigated variable star. After editing and acceptance of the light curve the last one becomes publicity accessed.

\section{Testing on real images}

The CoLiTecVS software (Pohorelov et al., 2016) was developed in continuous testing on the archival images taken on the Astronomical Observatory on Kolonica Sad- 
dle (Kudzej \& Dubovský, 2010) with several instruments. We have compared the results of differential photometry acquired in usual way with the new automatic reduction.

\subsection{Testing the influence of median filtering on the photometry results}

Important part of the reduction process in CoLiTecVS is the calibration of the images. The user can choose various combination of processes like bias correction, darkframe correction, flat-field correction and background brightness equalization by inverse median filter. We have already published the results of massive testing in Dubovský et al. (2017). The conclusion is that it can replace the master-flat application if there are no structures on the images background with size similar to the sizes of the stars. The ideal combination of calibration processes is: dark-frame, flat-field for removing the small structures and inverse median filtering for background equalization. If high quality flat-field is not available, then it is better to skip the flat-field calibration.

\subsection{Testing the photometry tool of the CoLiTecVS}

CoLiTecVS works as compact automatic toll. It takes raw images and provides light curves. It is not possible to cut one partial process and test it. But there is the possibility to take the images calibrated by CoLiTecVS and apply another photometry software on them. Exactly this was our approach. We have compared CoLiTecVS photometry of constant stars with the values obtained by conventional reduction process i.e. calibration and photometry performed with Muniwin software (Motl, 2009) and subsequently the ensemble photometry with MCV software (Kim et al., 2004). We have performed many comparisons with different instruments in different star fields. More than 100 time series were reduced. The typical result is shown on the Figure 3.

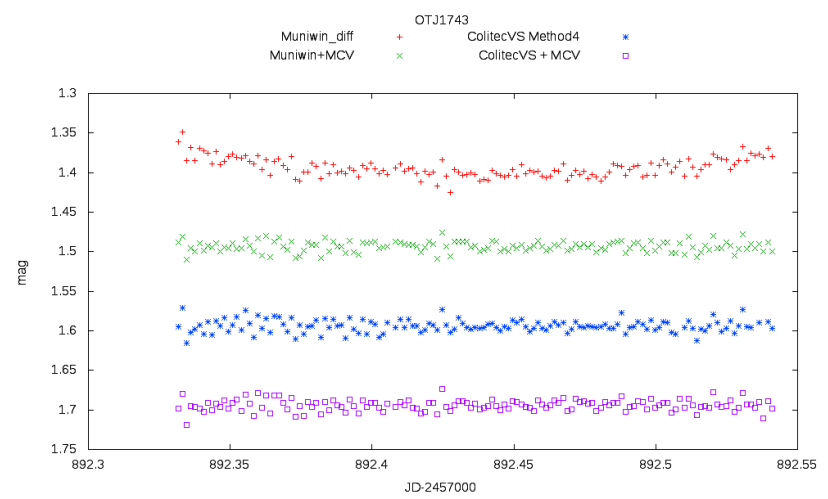

Figure 3: Comparison of photometry results of constant stars using different tools. The field of cataclysmic variable MASTER OT J174305.70+231107.8 was observed by Celestron 11" telescope equipped with MII G2-1600 CCD camera. The light curves are arbitrary shifted for clarity.

The meaning of the individual light curves is as follows: "Muniwin_diff" means single differential photometry with Muniwin software, one comparison star; "Muniwin + MCV" means ensemble photometry, instrumental magnitudes provided by Muniwin reduced by MCV software; "CoLiTecVS Method4" means ensemble photometry, fully automated output of CoLiTecVS; "CoLiTecVS + MCV" means ensemble photometry, instrumental magnitudes provided by CoLiTecVS reduced by MCV software.

The same result is presented also in numerical form in the Table 1.

Table 1: The means and standard deviations of the light curves depicted on the Figure 3.

\begin{tabular}{|l|c|c|}
\hline & Mean & $\begin{array}{c}\text { Standard } \\
\text { deviation }\end{array}$ \\
\hline Muniwin_diff & 1.3935 & 0.0118 \\
\hline Muniwin + MCV & 1.3935 & 0.0067 \\
\hline CoLiTecVS Method4 & 1.3939 & 0.0078 \\
\hline CoLiTecVS + MCV & 1.3941 & 0.0070 \\
\hline
\end{tabular}

The disadvantage of single comparison star is clearly visible. The trend with parabola shape is caused by extinction. The rest of the light curves are practically equal. So the CoLiTecVS can replace the conventional reduction process with the same precision and accuracy. If the user doesn't trust the automatic process completely, he has still the option to take instrumental output of CoLiTecVS and play with MCV. Especially removing the outstanding points on the light curves is still safer if doing manually.

\section{Conclusion}

New tool for automated reduction of photometric observations was developed. It includes the computational method for the brightness assessment of the investigated star, comparisons stars and light curve creation. The proposed method of inverse median filter application can be used for calibration of astronomical images without negative influence on the results of the photometry.

CoLiTecVS including VO technology is implemented in the Vihorlat Virtual Observatory (ViViO).

Acknowledgements. This work was supported by the Slovak Research and Development Agency under the contract No. APVV-15-0458.

\section{References}

Dubovský P.A., Briukhovetskyi O.B., Khlamov S.V. et al.: 2017, OEJV, 180, 16.

Kim Y., Andronov I.L., Jeon Y.B.: 2004, J. Astron. Space Science, 21, 191.

Kudzej I., Dubovský P.A.: 2010, Odessa Astron. Publ., 23, 70 .

Motl D.: 2009, C-Munipack, [online] Available at: (http://cmunipack.sourceforge.net).

Pohorelov A.V., Khlamov S.V. et al.: 2016, Odessa Astron. Publ., 29, 136.

Savanevych V.E. et al.: 2015, MNRAS, 451, 3287-3298.

Savanevych V.E. et al.: 2015, Kinem. and Phys. of Cel. Bod., 31, 302. 